\section{Perfil de pacientes submetidos à cirurgia bariátrica, assistidos pelo Sistema Único de Saúde do Brasil: revisão sistemática}

\author{
The profile of patients undergoing bariatric surgery \\ in the Brazilian Unified National Health System: \\ a systematic review
}

\author{
Perfil de los pacientes sometidos a cirugía \\ bariátrica, asistidos por el Sistema Único de Salud \\ en Brasil: revisión sistemática
}

\author{
${ }^{1}$ Hospital das Clínicas, \\ Universidade Federal de \\ Minas Gerais, Belo Horizonte, \\ Brasil. \\ 2 Faculdade de Medicina \\ Universidade Federal de \\ Minas Gerais, Belo Horizonte, \\ Brasil. \\ 3 Unimed-BH, Belo Horizonte, \\ Brasil. \\ Correspondência \\ S. M. B. Kelles \\ Núcleo de Avaliação de \\ Tecnologias em Saúde, \\ Hospital das Clínicas, \\ Universidade Federal de \\ Minas Gerais. \\ Av. Alfredo Balena 110 \\ Io andar, ala Oeste, \\ Belo Horizonte, $M G$ \\ 30130-100, Brasil. \\ silvanakelles@gmail.com
}

\begin{abstract}
Nearly one million Brazilians were morbidly obese in 2013. Bariatric surgery is an option for sustained weight loss, and the Brazilian Unified National Health System (SUS) had provided 50,000 such procedures as of 2014. The SUS database does not provide anthropometric and comorbidity data on these patients, so the aim of the current study was to perform a systematic review to assess the profile of SUS patients that underwent bariatric surgery from 1998 to 2014. The MEDLINE, LILACS, SciELO, and Scopus databases were searched, and the methodological quality of the included articles was assessed. Of the 1,591 identified studies, 39 were selected, 95\% of which were observational. Patients had a mean age of 41.4 years and mean body mass index of $48.6 \mathrm{~kg} / \mathrm{m}^{2} ; 21 \%$ were males, $61 \%$ hypertensive, $22 \%$ diabetics, and $31 \%$ presented sleep apnea. When compared to international study samples, SUS patients showed similar a anthropometric profile and comorbidities but higher prevalence of hypertension. The studies' low methodological quality suggests caution in interpreting the results.
\end{abstract}

Bariatric Surgery; Gastric Bypass; Health Profile
Silvana Marcia Bruschi Kelles 1,2,3

Maria de Fátima Haueisen Sander Diniz 2

Carla Jorge Machado 2

Sandhi Maria Barreto 2

\section{Resumo}

Em 2013, quase um milhão de brasileiros eram obesos graves. A cirurgia bariátrica é uma alternativa para a perda de peso sustentada. Até 2014 foram realizadas quase 50 mil cirurgias pelo Sistema Único de Saúde (SUS) do Brasil, mas dados antropométricos e de comorbidades destes pacientes são pouco conhecidos. O objetivo do estudo foi realizar uma revisão sistemática para conhecer o perfil do paciente submetido à cirurgia bariátrica pelo SUS até 2014. Foram consultadas as bases MEDLINE, LILACS, SciELO e Scopus. A qualidade metodológica dos artigos incluídos foi avaliada por escores de Carson e Newcastle-Ottawa. Foram identificados 1.591 estudos e selecionados 39, sendo 95\% observacionais. Em média, os pacientes têm 41,4 anos, índice de massa corporal 48,6kg/m², $21 \%$ são homens, $61 \%$ hipertensos, $22 \%$ diabéticos e $31 \%$ têm apneia do sono. Comparado com pacientes incluídos em estudos internacionais, o paciente SUS apresenta perfil antropométrico e de comorbidades semelhante, exceto pela maior prevalência de hipertensão. A baixa qualidade metodológica dos estudos sugere precaução na interpretação dos resultados.

Cirurgia Bariátrica; Derivação Gástrica; Perfil de Saúde 


\section{Introdução}

A prevalência de obesidade no Brasil tem aumentado ao longo dos anos, seguindo a tendência de países desenvolvidos. Segundo a Pesquisa de Orçamentos Familiares de 2008/2009 (POF 2008/2009) 1, o percentual de indivíduos obesos, índice de massa corporal (IMC) entre $35 \mathrm{e}<$ $40 \mathrm{~kg} / \mathrm{m}^{2}$, é de $2,8 \%$; obesos graves, IMC entre 40 e $<50 \mathrm{~kg} / \mathrm{m}^{2}$, são $0,7 \%$ e $0,04 \%$ de superobesos, com IMC $\geq 50 \mathrm{~kg} / \mathrm{m}^{2}$ (POF 2008/2009 dados não publicados). Em números absolutos, corrigidos para a população estimada para 2013, há cerca de um milhão de pessoas com obesidade grave no país, afetando principalmente as mulheres 1 .

As autoridades sanitárias brasileiras têm empreendido várias medidas para conter a progressão da obesidade para a obesidade grave ${ }^{2}$, mas existe hoje um grande contingente de indivíduos com IMC $\geq 40 \mathrm{~kg} / \mathrm{m}^{2}$. A obesidade grave é refratária à terapia dietética e medicamentosa, mas geralmente responde bem à cirurgia bariátrica 3 .

No Brasil, quase 150 milhões de indivíduos (75\% da população) dependem exclusivamente do Sistema Único de Saúde (SUS) para atenção à sua saúde. A cirurgia bariátrica está disponível no SUS desde o começo de 1999, no início exclusivamente em hospitais universitários 4 , passando a ser extensiva a centros públicos de atendimento em 2000 5. Atualmente são 96 hospitais especializados no SUS para a prestação de serviço de atenção à obesidade - tratamento cirúrgico, cirurgia reparadora e acompanhamento do paciente com obesidade, sendo 38 centros na Região Sudeste (Ministério da Saúde. Cadastro Nacional de Estabelecimentos de Saúde. http:// cnes.datasus.gov.br/Mod_Ind_Especialidades. asp? VEstado $=00 \&$ VMun $=00 \&$ VTerc $=00 \&$ VServi $\mathrm{co}=127 \& \mathrm{VClassificacao}=001 \& \mathrm{VAmbu}=\& \mathrm{VAmbu}$ SUS $=\& V H o s p=\& V H o s p S U S=1$, acessado em 31/ Ago/2013). Entre 2001 e 2014 foram realizadas 49.425 cirurgias bariátricas pelo SUS. Apesar disso, as filas de espera chegam a mais de três anos 6 . Devido à grande espera, é possível que os pacientes operados pelo SUS tenham um perfil pré-cirúrgico peculiar, com mais comorbidades e IMC mais alto.

Embora as publicações sobre cirurgia bariátrica sejam inúmeras na literatura internacional, existem poucos estudos nacionais sobre o assunto e, destes, raros envolvem pacientes atendidos pelo SUS. Sabe-se que algumas comorbidades são preditoras para complicações e mesmo óbito em pacientes submetidos à cirurgia bariátrica ${ }^{3}$. Conhecer o perfil antropométrico e de comorbidades dos pacientes atendidos pelo SUS é importante para dimensionar os riscos referentes à morbimortalidade da cirurgia e pro- visionar recursos, cuidados especiais e suporte pós-operatório.

O objetivo da presente revisão é descrever o perfil antropométrico e de comorbidades do paciente submetido à cirurgia bariátrica pelo SUS durante os últimos 15 anos.

\section{Material e métodos}

Foi conduzida uma busca extensiva na literatura publicada até dezembro de 2014, nas bases de dados MEDLINE (Medical Literature Analysis and Retrieval System Online) via PubMed, LILACS (Literatura Latino-Americana e do Caribe em Ciências da Saúde), SciELO (Scientific Electronic Library Online) e Scopus, com o objetivo de selecionar estudos que descrevessem o perfil dos pacientes submetidos à cirurgia bariátrica atendidos pelo SUS. Procedemos também à busca manual em bancos de teses, dissertações e monografias (Índice Bibliográfico Espanhol de Ciências da Saúde - IBECS e Coordenação de Aperfeiçoamento de Pessoal de Nível Superior - Capes) e na base de dados Coleciona SUS disponível na Biblioteca Virtual de Saúde (BVS). Adicionalmente, buscamos listas de referências dos artigos identificados e selecionados.

\section{Critérios de inclusão}

Foram selecionados artigos que descrevessem cirurgia bariátrica em pacientes oriundos de hospitais públicos ou universitários, com informações de dados antropométricos e, quando disponível, perfil de comorbidades. A descrição "via laparotômica" foi acrescida à estratégia de busca visando a torná-la mais específica. Dado que não existe um descritor para selecionar pacientes atendidos pelo SUS, e como somente a cirurgia por via laparotômica tem cobertura pelo sistema público, utilizamos esta via de acesso cirúrgico como proxy para a busca de artigos elegíveis. Foram selecionados artigos, teses, dissertações ou monografias que incluíssem pacientes operados a partir de 1999, época em que a cirurgia bariátrica teve cobertura pelo SUS (Portaria GM/ MS no 252 4, de 30 de março de 1999). Quando a origem ou o período de inclusão dos pacientes não estava descrita no texto, os pesquisadores entraram em contato com os autores dos artigos originais para solicitar este esclarecimento. Os estudos cuja informação sobre a origem ou período de inclusão não foi recuperada foram excluídos. Não houve restrição de data ou de idioma para a pesquisa. 


\section{Critérios de exclusão}

Foram excluídos os artigos envolvendo pacientes de clínicas particulares ou atendidos por convênios ou que incluíssem apenas subgrupos específicos de pacientes - só diabéticos ou só pacientes do sexo feminino, por exemplo. Foram excluídas as publicações duplicadas, artigos de revisão narrativa, assim como artigos de séries de casos com menos de vinte pacientes ou relatos de casos. Também foram excluídos trabalhos que avaliavam pacientes em fila de espera para a cirurgia bariátrica agendada no SUS, por considerar que seu perfil antropométrico e de comorbidades poderia mudar ao longo do tempo de espera, que pode ser de até três anos 6 .

As estratégias de busca utilizadas nas bases de dados MEDLINE via PubMed, LILACS, Scopus e SciELO, e o número de estudos encontrados e recuperados estão descritos na Tabela 1. Os documentos recuperados oriundos de teses, dissertações e monografias, assim como a busca de referências não encontradas por outros meios foram descritos como busca manual (diagrama PRISMA).

\section{Seleção dos artigos}

A seleção inicial dos artigos foi baseada na análise de título e resumo avaliados de forma independente por dois pesquisadores (S.M.B.K. e M.F.H.S.D.). A decisão sobre a inclusão ou não de artigos com avaliação controversa foi feita com base em consenso entre os dois autores. Os artigos selecionados foram lidos na íntegra e incluídos aqueles que respondiam à questão formulada para esta revisão.

\section{Avaliação da qualidade dos estudos}

A avaliação da qualidade metodológica de artigos observacionais é um grande desafio. $\mathrm{O}$ objetivo dessa avaliação é quantificar a validade interna e externa dos artigos. Após extensa busca na literatura, foram selecionados três instrumentos para a aferição da qualidade dos estudos incluídos nesta revisão: o formulário desenvolvido por Carson em 19947 para avaliação de estudos não randomizados e a Escala Newcastle-Ottawa (NOS) para estudos de coorte 8 , e sua adaptação para avaliação de estudos transversais 9 .

O formulário de Carson 7 é um instrumento com 10 itens, que avalia principalmente a identificação da coorte, sua descrição e o acompanhamento dos indivíduos. O instrumento, com escore máximo de qualidade de 10 pontos, foi originalmente desenvolvido para ser utilizado em avaliação de estudos prognósticos, com base nas recomendações da Universidade McMaster do Canadá. A NOS 8 tem sido a mais amplamente utilizada para avaliar a qualidade metodológica de estudos de casos e controles e de estudos de coorte quanto à amostragem, seleção, exposição e desfechos clínicos, sendo atribuída uma estre-

Tabela 1

Estratégia de busca e número de estudos selecionados e incluídos nesta revisão.

\begin{tabular}{|c|c|c|}
\hline Base de dados & Estratégia de busca & Resultado \\
\hline \multirow[t]{7}{*}{ MEDLINE via PubMed } & ((("bariatric surgery"[MeSH Terms] OR ("bariatric"[All Fields] AND "surgery"[All & 586 \\
\hline & Fields]) OR "bariatric surgery"[All Fields]) OR ("gastric bypass"[MeSH Terms] OR & \\
\hline & ("gastric"[All Fields] AND "bypass"[All Fields]) OR "gastric bypass"[All Fields] OR & \\
\hline & "roux en y gastric bypass"[All Fields])) AND (brasil[All Fields] OR ("brazil"[MeSH & \\
\hline & Terms] OR "brazil"[All Fields]))) NOT ("laparoscopy"[MeSH Terms] OR & \\
\hline & "laparoscopy"[All Fields] OR "laparoscopic"[All Fields]) AND ("1999/01/01"[PDAT]: & \\
\hline & "2014/12/31"[PDAT]) & \\
\hline \multirow[t]{3}{*}{ LILACS } & tw: (cirurgia bariátrica OR bariatric surgery OR gastric bypass OR derivação & 545 \\
\hline & gástrica) AND (instance:"regional") AND ( db:("LILACS") & \\
\hline & filtro data de publicação $\geq 1999$ & \\
\hline \multirow[t]{2}{*}{ SciELO } & (cirurgia bariátrica OR derivação gástrica) OR (gastric bypass OR bariatric surgery)) & 409 \\
\hline & Filtros Brasil ano de publicação 1999-2014 & \\
\hline \multirow[t]{2}{*}{ Scopus } & (bariatric surgery OR gastric bypass OR roux-en-y gastric bypass) AND & 46 \\
\hline & (brazil OR Brasil) AND PUBYEAR > 1999 & \\
\hline Busca manual (bancos de teses e dissertações, & & 5 \\
\hline referências de artigos incluídos) & & \\
\hline
\end{tabular}


la para cada item contemplado, resultando em escores que variam de 0 (pior) a 9 (melhor). $\mathrm{O}$ terceiro instrumento foi adaptado do original de Wells et al. ${ }^{8}$ por Herzog et al. ${ }^{9}$ para avaliação de estudos transversais. Pontua os estudos em uma escala entre 0 e 10, em que 10 corresponde à melhor qualidade metodológica.

Além desses escores, foi considerada como relevante para a avaliação da qualidade metodológica dos artigos a descrição dos critérios para a atribuição de comorbidades, ou seja, os parâmetros usados para considerar o paciente hipertenso, diabético, dislipidêmico ou outros. Esse item foi qualificado apenas como presente ou ausente.

Os estudos foram avaliados de forma independente por dois pesquisadores (S.M.B.K. e M.F.H.S.D.).

\section{Análise estatística}

Os dados de prevalência de comorbidades e distribuição por sexo, idade e IMC foram consolidados utilizando-se o modelo de efeito aleatório que considera tanto o tamanho da amostra como estima a variação interestudos (heterogeneidade). Foram computadas médias e desvios padrão para as variáveis contínuas e proporção e tamanho da amostra para as variáveis categóricas. $\mathrm{O}$ cálculo retornou a média e intervalo de $95 \%$ de confiança para cada variável contínua. Para as proporções, a transformação para logit foi aplicada para avaliação dos dados agregados (pooling). $\mathrm{O}$ resultado foi exponenciado em seguida, retornando, assim, para a forma de proporção, por ser uma apresentação mais fácil de interpretar. A heterogeneidade entre os estudos foi avaliada pelo I2 . A estatística I2 quantifica a inconsistência entre estudos para avaliar o impacto da heterogeneidade sobre a metanálise. Assim, I² próximo de 0 sugere que toda a dispersão pode ser atribuída ao erro aleatório do estudo. Valores da ordem de $25 \%, 50 \%$ e $75 \%$ devem ser considerados como baixa, moderada e alta heterogeneidade 10 .

Para selecionar subgrupos de estudos com menor heterogeneidade procedemos à análise avaliando três estratos: (1) todos os artigos incluídos; (2) apenas os artigos que atingissem, pelo menos, metade mais um do escore máximo possível; e (3) apenas os estudos que descrevessem os critérios para considerar o paciente como portador de comorbidades. Os dois ensaios clínicos randomizados encontrados foram considerados de boa qualidade metodológica e incluídos no grupo dos maiores escores. Para a análise de subgrupos, o modelo misto de análise do efeito foi utilizado, assumindo que a variância dentro dos estudos era a mesma para todos, e a combina- ção dos subgrupos foi avaliada por meio do seu efeito randômico. Não procedemos à análise de subgrupos que incluíam dois ou menos estudos.

Para todos os cálculos foi utilizado o software Comprehensive Meta-Analysis versão 2.2 (Biostat, Englewood, Estados Unidos; http:// www.meta-analysis.com/index.php).

\section{Resultados}

Um total de 1.591 artigos foi identificado com base nas estratégias da pesquisa, dos quais 115 potencialmente elegíveis foram recuperados para a leitura completa. Desses, 39 artigos atenderam os critérios de inclusão (Figura 1).

No total, foram incluídos na revisão dados de 3.845 pacientes. A Tabela 2 mostra as características dos estudos incluídos: autor, ano de publicação e periódico, desenho do estudo, local da pesquisa, período de recrutamento, número de pacientes, tipo de amostra, se apresentavam descrição dos critérios utilizados para aferição de comorbidades (quando pertinente), escore de Carson e NOS

Foram 37 (94,9\%) estudos observacionais, sendo nove $(23,1 \%)$ retrospectivos, $22(56,4 \%)$ prospectivos e seis $(15,4 \%)$ transversais. Dois eram ensaios clínicos randomizados $(5,1 \%)$. Vinte e nove publicações $(74,4 \%)$ provieram de pesquisas em hospitais universitários. Todas as amostras, exceto duas, foram amostras por conveniência. Todos os pacientes foram operados pela técnica de derivação gástrica em "Y-deRoux", laparotômica.

Dos 28 artigos que descreviam comorbidades dos pacientes operados, apenas sete (25\%) descreviam os parâmetros usados como diagnóstico de comorbidades.

A avaliação da qualidade metodológica dos estudos de coorte pelo escore de Carson mostrou média de 5,5 pontos (em máximo de 10 pontos) com desvio padrão de 1,3. A avaliação pelo NOS para estudos de coorte mostrou média de 5,4 estrelas (máximo de 9) e desvio padrão de 1,2. Para os estudos transversais, a média e desvio padrão pelo NOS foram de 6,2 e 1,2, respectivamente. Do total, 15 estudos $(48,4 \%)$ avaliados pela escala de Carson e 21 (56,8\%) pelo NOS, entre estudos de coorte e transversais, apresentaram escores iguais ou maiores que a média mais um.

Vinte e três artigos, totalizando 2.465 pacientes, apresentaram o perfil de pacientes submetidos à cirurgia bariátrica no Estado de São Paulo. Em Minas Gerais foram cinco artigos envolvendo 570 pacientes; quatro artigos no Paraná (total = 204 pacientes), três em Pernambuco (total = 262), dois no Rio Grande do Sul (total = 209), um 
Figura 1

Flugorama da seleção dos estudos.

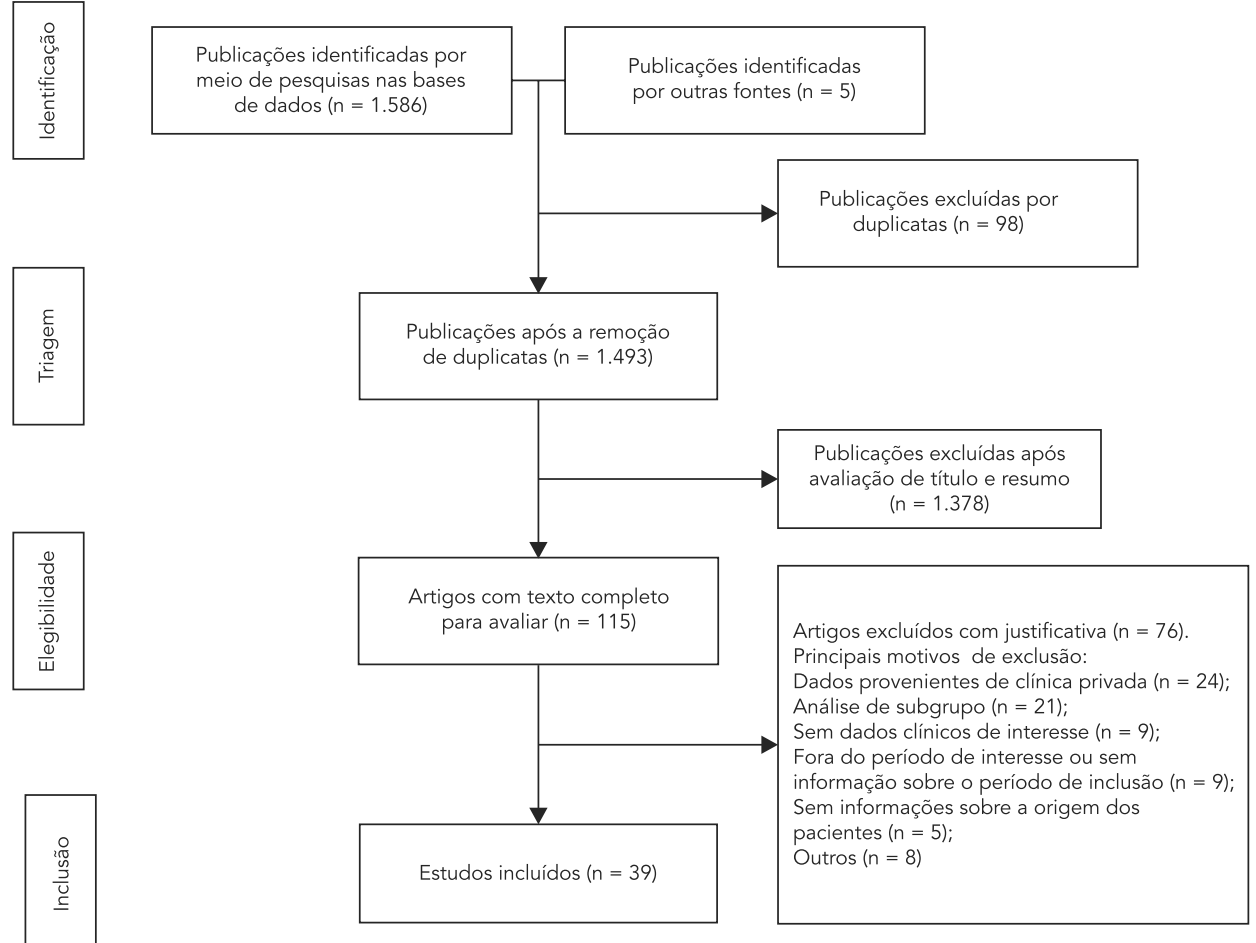

Adaptado de PRISMA (Preferred Reporting Items for Systematic Reviews and Meta-analysis; http://www.prisma-statement.org/ index.html).

Tabela 2

Descrição do estudo, desenho, local da pesquisa, número de participantes, tipo de amostra, presença de critérios para comorbidades, escores de avaliação de qualidade dos estudos.

\begin{tabular}{|c|c|c|c|c|c|c|c|c|}
\hline $\begin{array}{l}\text { Referência } \\
\text { (ano) }\end{array}$ & $\begin{array}{l}\text { Desenho do } \\
\text { estudo * }\end{array}$ & $\begin{array}{l}\text { Local da pesquisa } \\
\text { (Estado) }\end{array}$ & Período & $\mathbf{N} * *$ & $\begin{array}{c}\text { Tipo de } \\
\text { amostra *** }\end{array}$ & $\begin{array}{l}\text { Critérios para } \\
\text { comorbidades \# }\end{array}$ & $\begin{array}{l}\text { Escore de } \\
\text { Carson \#\# }\end{array}$ & NOS \#\#\# \\
\hline Abreu et al. 27 (2007) & OP & $\begin{array}{l}\text { Hospital Regional de } \\
\text { Assis (São Paulo) }\end{array}$ & $2003-2005$ & 20 & C & N & 7 & 4 \\
\hline $\begin{array}{l}\text { Anderi Jr. et al. } 28 \\
\text { (2007) }\end{array}$ & OP & $\begin{array}{l}\text { Faculdade de } \\
\text { Medicina do ABC } \\
\text { (São Paulo) }\end{array}$ & $2000-2006$ & 312 & C & N & 6 & 2 \\
\hline $\begin{array}{l}\text { Blume et al. } 29 \\
(2012)\end{array}$ & OR & $\begin{array}{l}\text { Hospital São Lucas/ } \\
\text { PUCRS } \\
\text { (Rio Grande do Sul) }\end{array}$ & $2000-2005$ & 170 & C & N & 6 & 6 \\
\hline $\begin{array}{l}\text { Cardozo et al. } 30 \\
\text { (2014) }\end{array}$ & OP & $\begin{array}{l}\text { Hospital Nossa } \\
\text { Senhora da } \\
\text { Conceição } \\
\text { (Rio Grande do Sul) }\end{array}$ & 2009-2011 & 39 & C & N & 7 & 6 \\
\hline
\end{tabular}

(continua) 
Tabela 2 (continuação)

\begin{tabular}{|c|c|c|c|c|c|c|c|c|}
\hline $\begin{array}{l}\text { Referência } \\
\text { (ano) }\end{array}$ & $\begin{array}{c}\text { Desenho do } \\
\text { estudo * }\end{array}$ & $\begin{array}{l}\text { Local da pesquisa } \\
\text { (Estado) }\end{array}$ & Período & $\mathbf{N} * \star$ & $\begin{array}{c}\text { Tipo de } \\
\text { amostra } \star \star \star\end{array}$ & $\begin{array}{l}\text { Critérios para } \\
\text { comorbidades \# }\end{array}$ & $\begin{array}{l}\text { Escore de } \\
\text { Carson \#\# }\end{array}$ & NOS \#\#\# \\
\hline $\begin{array}{l}\text { Carneiro et al. } 31 \\
\text { (2012) }\end{array}$ & OP & $\begin{array}{c}\text { Centro Clínico de } \\
\text { Obesidade/UNIFESP } \\
\text { (São Paulo) }\end{array}$ & 2004-2007 & 132 & C & $S$ & 6 & 5 \\
\hline $\begin{array}{l}\text { Cazzo et al. } 32 \\
(2014)\end{array}$ & OP & $\begin{array}{l}\text { Hospital das Clínicas/ } \\
\text { Unicamp (São Paulo) }\end{array}$ & 2011-2012 & 63 & C & $\mathrm{N}$ & 5 & 6 \\
\hline $\begin{array}{l}\text { Costa et al. } 33 \\
(2014)\end{array}$ & OP & $\begin{array}{l}\text { Hospital do Servidor } \\
\text { Público Estadual } \\
\text { (São Paulo) }\end{array}$ & 2007-2008 & 143 & C & $N$ & 5 & 8 \\
\hline $\begin{array}{l}\text { Costa-Matos et al. } 34 \\
\text { (2009) }\end{array}$ & OR & $\begin{array}{l}\text { Santa Casa de São } \\
\text { Paulo (São Paulo) }\end{array}$ & 2000-2005 & 58 & C & $\mathrm{N}$ & 6 & 6 \\
\hline $\begin{array}{l}\text { Cunha et al. } 35 \\
\text { (2006) }\end{array}$ & OP & $\begin{array}{l}\text { Hospital de Clínicas/ } \\
\text { UFPR (Paraná) }\end{array}$ & 1999-2000 & 23 & C & $N$ & 5 & 5 \\
\hline $\begin{array}{l}\text { Diniz et al. } 36 \\
\text { (2009) }\end{array}$ & OP & $\begin{array}{c}\text { Hospital das Clinicas } \\
\text { da UFMG } \\
\text { (Minas Gerais) }\end{array}$ & 1998-2005 & 193 & C & $\mathrm{S}$ & 5 & 6 \\
\hline $\begin{array}{l}\text { Donadelli et al. } 37 \\
\text { (2011) }\end{array}$ & OP & $\begin{array}{l}\text { Hospital Escola da } \\
\text { Universidade de } \\
\text { Ribeirão Preto } \\
\text { (São Paulo) }\end{array}$ & 2001-2007 & 42 & C & $\mathrm{S}$ & 5 & 5 \\
\hline $\begin{array}{l}\text { Duarte-Guerra et } \\
\text { al. } 11 \text { (2014) }\end{array}$ & OP & $\begin{array}{c}\text { Hospital } \\
\text { Universitário/USP } \\
\text { (São Paulo) }\end{array}$ & 2010 & 393 & C & NA & 7 & 6 \\
\hline $\begin{array}{l}\text { Franca } 38 \\
(2011)\end{array}$ & $\mathrm{T}$ & $\begin{array}{l}\text { Hospital do Servidor } \\
\text { Público Municipal } \\
\text { (São Paulo) }\end{array}$ & 2009-2010 & 21 & C & NA & NA & 7 \\
\hline $\begin{array}{l}\text { Freire et al. } 39 \\
(2012)\end{array}$ & $\mathrm{T}$ & $\begin{array}{c}\text { Hospital das Clínicas } \\
\text { da UFMG } \\
\text { (Minas Gerais) }\end{array}$ & 1998-2008 & 100 & C & $\mathrm{N}$ & NA & 6 \\
\hline $\begin{array}{l}\text { Freitas } 40 \\
(2011)\end{array}$ & OP & $\begin{array}{c}\text { Hospital Universitário } \\
\text { Onofre Lopes (Rio } \\
\text { Grande do Norte) }\end{array}$ & $\begin{array}{l}\text { Fev/2009- } \\
\text { Dez/2010 }\end{array}$ & 39 & C & $N$ & 5 & 5 \\
\hline $\begin{array}{l}\text { Freitas Junior et } \\
\text { al. } 41 \text { (2014) }\end{array}$ & OP & $\begin{array}{l}\text { Santa Casa de São } \\
\text { Paulo (São Paulo) }\end{array}$ & 2009 & 36 & C & NA & 4 & 6 \\
\hline $\begin{array}{l}\text { Jóia Neto et al. } 42 \\
(2010)\end{array}$ & OP & $\begin{array}{c}\text { Hospital da } \\
\text { Universidade } \\
\text { Estadual de Londrina } \\
\text { (Paraná) }\end{array}$ & 2001-2007 & 44 & C & $\mathrm{N}$ & 5 & 6 \\
\hline $\begin{array}{l}\text { Khawali et al. } 6 \\
\text { (2012) }\end{array}$ & $\mathrm{T}$ & $\begin{array}{c}\text { Centro de } \\
\text { Obesidade/UNIFESP } \\
\text { (São Paulo) }\end{array}$ & 1999-2005 & 125 & C & $\mathrm{N}$ & NA & 7 \\
\hline $\begin{array}{l}\text { Kuga et al. } 43 \\
\text { (2007) }\end{array}$ & OR & $\begin{array}{l}\text { Escola de Medicina/ } \\
\text { USP (São Paulo) }\end{array}$ & 2004-2005 & 40 & C & NA & 4 & 4 \\
\hline $\begin{array}{l}\text { Laurino Neto et } \\
\text { al. } 44 \text { (2012) }\end{array}$ & OR & $\begin{array}{l}\text { Hospital de Ensino } \\
\text { de Mandaqui } \\
\text { (São Paulo) }\end{array}$ & 1998-2005 & 140 & C & S & 7 & 5 \\
\hline $\begin{array}{l}\text { Lima et al. } 45 \\
(2005)\end{array}$ & $\mathrm{T}$ & $\begin{array}{c}\text { Hospital das Clínicas } \\
\text { da UFMG } \\
\text { (Minas Gerais) }\end{array}$ & $2000-2003$ & 112 & C & $\mathrm{N}$ & NA & 7 \\
\hline
\end{tabular}

(continua) 
Tabela 2 (continuação)

\begin{tabular}{|c|c|c|c|c|c|c|c|c|}
\hline $\begin{array}{l}\text { Referência } \\
\text { (ano) }\end{array}$ & $\begin{array}{l}\text { Desenho do } \\
\text { estudo * }\end{array}$ & $\begin{array}{l}\text { Local da pesquisa } \\
\text { (Estado) }\end{array}$ & Período & $\mathbf{N} * \star$ & $\begin{array}{c}\text { Tipo de } \\
\text { amostra } * \star \star\end{array}$ & $\begin{array}{l}\text { Critérios para } \\
\text { comorbidades \# }\end{array}$ & $\begin{array}{l}\text { Escore de } \\
\text { Carson \#\# }\end{array}$ & NOS \#\#\# \\
\hline $\begin{array}{l}\text { Machado } 46 \\
\text { (2008) }\end{array}$ & OP & $\begin{array}{l}\text { Hospital das Clínicas/ } \\
\text { Faculdade de } \\
\text { Medicina da USP } \\
\text { (São Paulo) }\end{array}$ & $2002-2008$ & 50 & C & NA & 6 & 4 \\
\hline $\begin{array}{l}\text { Moreira et al. } 47 \\
\text { (2010) }\end{array}$ & $\mathrm{T}$ & $\begin{array}{l}\text { Hospital universitário } \\
\text { (Pernambuco) }\end{array}$ & $\begin{array}{c}\text { Mai- } \\
\text { Nov/2007 }\end{array}$ & 37 & C & $\mathrm{N}$ & NA & 6 \\
\hline $\begin{array}{l}\text { Moreira et al. } 12 \\
(2013)\end{array}$ & $\mathrm{T}$ & $\begin{array}{l}\text { Hospital público em } \\
\text { Fortaleza (Ceará) }\end{array}$ & $\begin{array}{c}\text { Abr- } \\
\text { Ago/2009 }\end{array}$ & 20 & C & $\mathrm{N}$ & NA & 4 \\
\hline $\begin{array}{l}\text { Oliveira } 48 \\
\text { (2007) }\end{array}$ & OR & $\begin{array}{l}\text { Hospital público em } \\
\text { São Paulo } \\
\text { (São Paulo) }\end{array}$ & $\begin{array}{l}\text { Jan/2001- } \\
\text { Mar/2007 }\end{array}$ & 106 & C & $\mathrm{N}$ & 3 & 6 \\
\hline $\begin{array}{l}\text { Oliveira et al. } 13 \\
\text { (2009) }\end{array}$ & OP & $\begin{array}{c}\text { Hospital das Clínicas } \\
\text { da UFMG } \\
\text { (Minas Gerais) }\end{array}$ & 2005-2006 & 22 & C & $N$ & 6 & 6 \\
\hline $\begin{array}{l}\text { Paisani et al. } 49 \\
\text { (2005) }\end{array}$ & OP & $\begin{array}{l}\text { Hospital São Paulo/ } \\
\text { UNIFESP (São Paulo) }\end{array}$ & 2001-2002 & 21 & C & NA & 5 & 7 \\
\hline $\begin{array}{l}\text { Pedrosa et al. } 50 \\
\text { (2009) }\end{array}$ & OR & $\begin{array}{c}\text { Hospital } \\
\text { Universitário/UFPE } \\
\text { (Pernambuco) }\end{array}$ & $2002-2006$ & 205 & C & $\mathrm{N}$ & 4 & 5 \\
\hline $\begin{array}{l}\text { Pinheiro-Junior et } \\
\text { al. } 51 \text { (2012) }\end{array}$ & OP & $\begin{array}{l}\text { Hospital de Base de } \\
\text { São José do } \\
\text { Rio Preto (São Paulo) }\end{array}$ & $2000-2008$ & 220 & C & $\mathrm{S}$ & 7 & 7 \\
\hline $\begin{array}{l}\text { Porto } 52 \\
(2012)\end{array}$ & OR & $\begin{array}{l}\text { Hospital do Servidor } \\
\text { Público Municipal de } \\
\text { São Paulo } \\
\text { (São Paulo) }\end{array}$ & 2001-2005 & 85 & C & $\mathrm{N}$ & 4 & 5 \\
\hline $\begin{array}{l}\text { Salgado Jr. et al. } 53 \\
\text { (2010) }\end{array}$ & OP & $\begin{array}{l}\text { Hospital Escola da } \\
\text { Universidade de } \\
\text { Ribeirão Preto } \\
\text { (São Paulo) }\end{array}$ & $2007-2008$ & 107 & C & NA & 6 & 6 \\
\hline $\begin{array}{l}\text { Salgado Jr. et al. } 54 \\
\text { (2011) }\end{array}$ & ECR & $\begin{array}{c}\text { Faculdade de } \\
\text { Medicina de Ribeirão } \\
\text { Preto/USP } \\
\text { (São Paulo) }\end{array}$ & 2009-2010 & 40 & A & NA & NA & NA \\
\hline $\begin{array}{l}\text { Scabim et al. } 55 \\
\text { (2012) }\end{array}$ & OR & $\begin{array}{l}\text { Hospital das Clínicas/ } \\
\text { USP (São Paulo) }\end{array}$ & 2006-2008 & 241 & C & NA & 9 & 6 \\
\hline $\begin{array}{l}\text { Silva et al. } 56 \\
(2007)\end{array}$ & OP & $\begin{array}{l}\text { Hospital das Clínicas/ } \\
\text { Unicamp (São Paulo) }\end{array}$ & $2000-2002$ & 50 & C & NA & 4 & 4 \\
\hline $\begin{array}{l}\text { Silva \& Sanches } 57 \\
(2006)\end{array}$ & OR & $\begin{array}{c}\text { Santa Casa de Belo } \\
\text { Horizonte } \\
\text { (Minas Gerais) }\end{array}$ & 1999-2003 & 143 & C & $\mathrm{S}$ & 4 & 3 \\
\hline $\begin{array}{l}\text { Silva et al. } 58 \\
(2013)\end{array}$ & OP & $\begin{array}{c}\text { Hospital } \\
\text { Universitário/UFAL } \\
\text { (Alagoas) }\end{array}$ & $2005-2010$ & 96 & C & $\mathrm{S}$ & 6 & 5 \\
\hline $\begin{array}{l}\text { Valezi \& Machado } 14 \\
\text { (2011) }\end{array}$ & OP & $\begin{array}{c}\text { Hospital Escola } \\
\text { da Universidade } \\
\text { Estadual de Londrina } \\
\text { (Paraná) }\end{array}$ & 2007 & 43 & C & $\mathrm{N}$ & 5 & 5 \\
\hline
\end{tabular}

(continua) 
Tabela 2 (continuação)

\begin{tabular}{|c|c|c|c|c|c|c|c|c|}
\hline $\begin{array}{l}\text { Referência } \\
\text { (ano) }\end{array}$ & $\begin{array}{c}\text { Desenho do } \\
\text { estudo * }\end{array}$ & $\begin{array}{l}\text { Local da pesquisa } \\
\text { (Estado) }\end{array}$ & Período & $N * *$ & 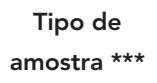 & $\begin{array}{l}\text { Critérios para } \\
\text { comorbidades \# }\end{array}$ & $\begin{array}{l}\text { Escore de } \\
\text { Carson \#\# }\end{array}$ & NOS \#\#\# \\
\hline Valezi et al. 59 & ECR & Hospital da & 2009-2011 & 94 & A & $\mathrm{N}$ & NA & NA \\
\hline \multirow[t]{3}{*}{ (2014) } & & Universidade & & & & & & \\
\hline & & Estadual de Londrina & & & & & & \\
\hline & & (Paraná) & & & & & & \\
\hline Xavier et al. 60 & OP & Faculdade de & 2006 & 20 & C & NA & 7 & 6 \\
\hline \multirow[t]{3}{*}{ (2010) } & & Medicina de Ribeirão & & & & & & \\
\hline & & Preto/USP & & & & & & \\
\hline & & (São Paulo) & & & & & & \\
\hline
\end{tabular}

PUCRS: Pontifícia Universidade Católica do Rio Grande do Sul; UFAL: Universidade Federal de Alagoas; UFMG: Universidade Federal de Minas Gerais; UFPE: Universidade Federal de Pernambuco; UFPR: Universidade Federal do Paraná; Unicamp: Universidade Estadual de Campinas; UNIFESP: Universidade Federal de São Paulo.

* Desenho do estudo: $E C R$ = Ensaio clínico randomizado; OP = observacional prospectivo; OR = observacional retrospectivo; $T$ = transversal;

** $\mathrm{N}=$ número de pacientes incluídos no estudo;

*** Tipo de amostra: $\mathrm{A}=$ Aleatorizada; $\mathrm{C}=$ Conveniência;

\# Critérios para aferição de comorbidades: $\mathrm{N}$ = não descrito; NA = Não se aplica/Não há descrição de comorbidades; $\mathrm{S}=$ descrito;

\#\# Escore de Carson: escore máximo (melhor qualidade metodológica) = 10;

\#\#\# NOS (Escala Newcastle-Ottawa): (a) para estudos de coorte - escore máximo 9; (b) para estudos transversais - escore máximo 10.

em Alagoas (96) e um do Rio Grande do Norte (39 pacientes). Os estudos incluídos, totalizando 3.845 pacientes, representam menos de $10 \%$ dos 49.425 pacientes submetidos à cirurgia bariátrica no Brasil no período (dado disponível no DATASUS; http://datasus.saude.gov.br/). Verificamos ainda que não foram encontrados estudos que atendessem os critérios de inclusão para pacientes operados nas regiões Norte e Centrooeste, e que mais de três quartos dos pacientes incluídos na presente revisão foram operados na Região Sudeste. Finalmente, não há informação se as amostras estudadas nas publicações selecionadas são representativas da totalidade dos pacientes operados no estado ou serviço(s) durante o período de estudo, ou mesmo se há superposição entre os pacientes incluídos em estudos realizados dentro de um mesmo estado. A Tabela 3 mostra o total de cirurgias bariátricas realizadas pelo SUS entre 2000 e 2014 e o percentual da população de operados incluída nos estudos, por região do Brasil.

A média de idade entre os pacientes incluídos nos estudos variou entre 32,3 ( $\mathrm{DP}=8,9$ ) anos e $48,3(\mathrm{DP}=10,2)$ anos. O percentual de pacientes do sexo masculino variou de $2,5 \%$ a $48,2 \%$. A média de IMC alternou entre $37,4 \mathrm{~kg} / \mathrm{m}^{2}(\mathrm{DP}=3,0) \mathrm{e}$ $56,8 \mathrm{~kg} / \mathrm{m}^{2}(\mathrm{DP}=5,5)$. Vinte e três estudos $(59 \%)$ apresentavam informações sobre presença de hipertensão, com percentual de portadores variando entre $35,9 \%$ e $86,5 \%$. Vinte e três estudos (59\%) apresentavam informações sobre diabetes, com prevalência mínima de $10 \%$ e máxima de
44,8\%. Presença de dislipidemia foi descrita em 17 dos estudos avaliados, artropatia foi descrita em seis, apneia do sono em seis e tabagismo em quatro. As comorbidades, por estudo, estão descritas na Tabela 4.

Quatro artigos 11,12,13,14 apresentavam o percentual de superobesos (IMC $>50 \mathrm{~kg} / \mathrm{m}^{2}$ ) na amostra, com frequência variando entre $20 \%$ e $64 \%$.

A síntese dos dados antropométricos e de perfil de comorbidades (Tabela 5), ponderada pelo tamanho das amostras, mostrou idade média de 41,4 anos, IMC médio de $48,6 \mathrm{~kg} / \mathrm{m}^{2} \mathrm{e}$

Tabela 3

Número e percentual de pacientes submetidos à cirurgia bariátrica pelo Sistema Único de Saúde (SUS) entre 2000 e 2014, representados nos estudos incluídos na revisão, por região do Brasil.

\begin{tabular}{lcc}
\hline Região & $\begin{array}{c}\text { Cirurgias } \\
\text { realizadas }\end{array}$ & $\begin{array}{c}\text { Pacientes } \\
\text { representados nos } \\
\text { estudos incluídos (\%) }\end{array}$ \\
\hline Norte & 942 & $0(0,0)$ \\
Nordeste & 4.619 & $397(8,6)$ \\
Centro-oeste & 1.560 & $0(0,0)$ \\
Sudeste & 19.344 & $3.035(15,7)$ \\
Sul & 22.960 & $413(1,8)$ \\
Total & 49.425 & $3.845(7,8)$ \\
\hline
\end{tabular}


Tabela 4

Descrição dos dados obtidos nos artigos incluídos na revisão, com o número de participantes e descrição antropométrica e de comorbidades.

\begin{tabular}{|c|c|c|c|c|c|c|c|c|c|}
\hline $\begin{array}{l}\text { Referência } \\
\text { (ano) }\end{array}$ & $\begin{array}{l}\text { Idade em } \\
\text { anos } \\
\text { Média (DP) }\end{array}$ & $\begin{array}{c}\text { Sexo } \\
\text { masculino } \\
N(\%)\end{array}$ & $\begin{array}{c}\text { IMC } \\
\text { Média (DP) }\end{array}$ & $\begin{array}{l}\text { Hipertensão } \\
\text { N (\%) }\end{array}$ & $\begin{array}{c}\text { Diabetes } \\
\text { N (\%) }\end{array}$ & $\begin{array}{l}\text { Artropatia } \\
\qquad N(\%)\end{array}$ & $\begin{array}{l}\text { Apneia } \\
\mathrm{N}(\%)\end{array}$ & $\begin{array}{c}\text { Dislipidemia } \\
\text { N (\%) }\end{array}$ & $\begin{array}{c}\text { Tabagismo } \\
\text { N (\%) }\end{array}$ \\
\hline Abreu et al. 27 (2007) & $36,4(12,3)$ & $7(35,0)$ & $\begin{array}{c}48,4 \\
\text { (IC95\%: } \\
44,3-52,4)\end{array}$ & & & & & $10(50,0)$ & \\
\hline Anderi Jr. et al. 28 (2007) & $\begin{array}{c}\text { Masculino: } \\
33 ; \\
\text { feminino: } \\
35\end{array}$ & $64(20,5)$ & $\begin{array}{c}\text { Masculino: } \\
42,5 ; \\
\text { feminino: } \\
47,5\end{array}$ & $112(35,9)$ & $29(9,3)$ & $43(13,8)$ & $62(19,9)$ & & \\
\hline Blume et al. 29 (2012) & $39,5(10,8)$ & $34(20,0)$ & $48,8(9,0)$ & & $25(14,7)$ & & & $93(54,7)$ & \\
\hline Cardozo et al. 30 (2014) & $45,7(9,5)$ & $1(2,6)$ & $50,7(5,9)$ & $29(74,4)$ & $16(41,0)$ & & & & \\
\hline Carneiro et al. 31 (2012) & $\begin{array}{l}\mathrm{G} 1 \mathrm{n}=85: \\
45,3(10,9) \\
\mathrm{G} 2 \mathrm{n}=47 \\
38,9(12,0)\end{array}$ & $\begin{array}{l}41(48,2) \\
12(25,5)\end{array}$ & $\begin{array}{l}48,8(7,3) \\
45,7(6,5)\end{array}$ & & & & $85(64,4)$ & & \\
\hline Cazzo et al. 32 (2014) & $40,7(10,3)$ & $13(20,6)$ & $37,4(3,0)$ & $41(65,1)$ & $27(42,8)$ & & & $23(36,5)$ & \\
\hline Costa et al. 33 (2014) & $\begin{array}{c}\mathrm{G} 1 \mathrm{n}=30: \\
39,3(10,3) \\
\mathrm{G} 2 \mathrm{n}=26: \\
45,0(11,3) \\
\mathrm{G} 3 \mathrm{n}=30: \\
46,7(9,7) \\
\mathrm{G} 4 \mathrm{n}=27 \\
48,3(10,2) \\
\mathrm{G} 5 \mathrm{n}=30: \\
45,7(7,1)\end{array}$ & $\begin{array}{c}4(13,3) \\
2(7,7) \\
1(3,3) \\
4(14,8) \\
3(10,0)\end{array}$ & $\begin{array}{l}45,5(5,3) \\
45,7(7,9) \\
45,1(5,8) \\
46,8(6,8) \\
47,7(5,2)\end{array}$ & $66(46,2)$ & $34(23,8)$ & $40(28,0)$ & $21(14,7)$ & $14(9,8)$ & \\
\hline $\begin{array}{l}\text { Costa-Matos et al. } 34 \\
\text { (2009) }\end{array}$ & $39,3(10,8)$ & $10(17,0)$ & $48,9(6,1)$ & $35(60,0)$ & $11(19,0)$ & & & & \\
\hline Cunha et al. 35 (2006) & $39,7(8,4)$ & $4(17,0)$ & $48,8(8,8)$ & $19(82,6)$ & & & & & \\
\hline Diniz et al. 36 (2009) & $38,7(9,0)$ & $51(26,4)$ & $52,0(8,1)$ & $122(63,2)$ & $46(23,8)$ & & & $95(49,2)$ & $33(17,1)$ \\
\hline Donadelli et al. 37 (2011) & $43,5(8,6)$ & $6(14,3)$ & $52,5(7,6)$ & $34(81,0)$ & $14(33,0)$ & & & $40(95,0)$ & $8(19,0)$ \\
\hline $\begin{array}{l}\text { Duarte-Guerra et al. } 11 \\
\text { (2014) }\end{array}$ & $43,0(11,5)$ & $82(20,9)$ & $47,8(7,5)$ & & & & & & \\
\hline Franca 38 (2011) & $44,8(6,5)$ & $5(23,8)$ & $44,9(4,9)$ & $16(76,2)$ & $6(28,6)$ & & & $2(9,5)$ & \\
\hline Freire et al. 39 (2012) & $45,1(9,9)$ & $16(16,0)$ & $54,9(8,8)$ & & & & & & \\
\hline Freitas 40 (2011) & $35,9(11,0)$ & $10(23,7)$ & $47,9(5,6)$ & $26(66,7)$ & $10(25,6)$ & & & & \\
\hline $\begin{array}{l}\text { Freitas Junior et al. } 41 \\
\text { (2014) }\end{array}$ & - & $7(19,4)$ & $50,8(7,7)$ & & & & & & \\
\hline Jóia Neto et al. 42 (2010) & $41,1(9,2)$ & $10(22,7)$ & $\begin{array}{c}\text { Mediana } \\
49,3\end{array}$ & & $13(29,6)$ & & & $4(9,1)$ & \\
\hline Khawali et al. 6 (2012) & $43,6(10,4)$ & $52(41,5)$ & $52,3(8,3)$ & $93(73,1)$ & $45(36,0)$ & $79(63,0)$ & $77(61,5)$ & $42(34,0)$ & \\
\hline Kuga et al. 43 (2007) & $44,5(10,0)$ & $6(15,0)$ & $53,3(8,2)$ & & & & & & \\
\hline $\begin{array}{l}\text { Laurino Neto et al. } 44 \\
\text { (2012) }\end{array}$ & $41,4(10,6)$ & $18(12,9)$ & $52,5(7,9)$ & $88(63,0)$ & $26(18,0)$ & $90(64,0)$ & $83(59,0)$ & $18(13,0)$ & \\
\hline Lima et al. 45 (2005) & $39,6(10,1)$ & $29(25,9)$ & $48,8(7,5)$ & $76(67,9)$ & $31(27,7)$ & $62(55,4)$ & & $45(40,2)$ & \\
\hline Machado 46 (2008) & $42,4(10,0)$ & $7(14)$ & $48,5(7,4)$ & & & & & & \\
\hline Moreira et al. 47 (2010) & Mediana 40 & $11(29,7)$ & $49,3(7,3)$ & $32(86,5)$ & $5(13,5)$ & & & $31(83,8)$ & \\
\hline Moreira et al. 12 (2013) & $32,3(8,9)$ & $8(40,0)$ & $45,2(7,6)$ & $9(45,0)$ & $2(10,0)$ & & & $19(95,0)$ & $8(40,0)$ \\
\hline Oliveira 48 (2007) & 48,6 & $19(17,9)$ & 48,7 & $71(66,9)$ & $15(14,1)$ & $6(5,6)$ & $3(2,8)$ & $7(6,6)$ & \\
\hline
\end{tabular}

(continua) 
Tabela 4 (continuação)

\begin{tabular}{|c|c|c|c|c|c|c|c|c|c|}
\hline $\begin{array}{l}\text { Referência } \\
\text { (ano) }\end{array}$ & $\begin{array}{l}\text { Idade em } \\
\text { anos } \\
\text { Média (DP) }\end{array}$ & $\begin{array}{c}\text { Sexo } \\
\text { masculino } \\
N(\%)\end{array}$ & Média (DP) & N (\%) & N (\%) & $\begin{array}{l}\text { Artropatia } \\
\text { N (\%) }\end{array}$ & $\begin{array}{l}\text { Apneia } \\
\text { N (\%) }\end{array}$ & Dislipidemia & N (\%) \\
\hline Oliveira et al. 13 (2009) & $39,9(11,2)$ & $8(36,0)$ & $52,4(8,0)$ & $14(64,0)$ & $3(14,0)$ & & & & \\
\hline Paisani et al. 49 (2005) & $39,0(9,7)$ & $3(14,2)$ & $50,4(7,6)$ & & & & & & \\
\hline Pedrosa et al. 50 (2009) & $38,4(9,6)$ & $58(28,8)$ & $48,6(9,0)$ & $108(52,7)$ & $24(11,7)$ & & & & \\
\hline Pinheiro-Junior et al. 51 & $\mathrm{G} 1 \mathrm{n}=114$ & $20(17,5)$ & $50,2(12,1)$ & $47(41,2)$ & $15(13,2)$ & & & $42(36,8)$ & \\
\hline (2012) & $\begin{array}{c}46,6(11,2) \\
G 2 n=106: \\
40,6(9,7)\end{array}$ & $21(19,8)$ & $47,9(14,2)$ & $60(56,6)$ & $30(28,3)$ & & & $39(36,8)$ & \\
\hline Porto 52 (2012) & $42,7(10,4)$ & $9(10,5)$ & $48,3(6,3)$ & $52(61,2)$ & $15(17,6)$ & & & & \\
\hline $\begin{array}{l}\text { Salgado Jr. et al. } 53 \\
(2010)\end{array}$ & $38,3(9,39)$ & $17(15,9)$ & $50,6(7,8)$ & & & & & & \\
\hline Salgado Jr. et al. 54 & $\mathrm{G} 1 \mathrm{n}=20$ & $2(10,0)$ & $44,3(5,0)$ & & & & & & \\
\hline (2011) & $\begin{array}{c}40,4(10,2) \\
G 2 n=20: \\
39,5(9,6)\end{array}$ & $5(25,0)$ & $46,6(7,2)$ & & & & & & \\
\hline Scabim et al. 55 (2012) & $44,4(11,6)$ & $46(19,1)$ & $47,2(6,2)$ & & & & & & \\
\hline Silva et al. 56 (2007) & $36,2(8,3)$ & $11(22)$ & $56,4(10,6)$ & & & & & & \\
\hline Silva \& Sanches 57 (2006) & 38,8 & $36(25,2)$ & $45,1(6,1)$ & & & & & $69(48,3)$ & \\
\hline Silva et al. 58 (2013) & $37,2(8,4)$ & $9(9,3)$ & $48,1(7,0)$ & $51(53,1)$ & $27(28,1)$ & & & $20(20,8)$ & \\
\hline $\begin{array}{l}\text { Valezi \& Machado } 14 \\
\text { (2011) }\end{array}$ & $35,9(12,2)$ & $12(27,9)$ & $41,8(4,4)$ & $16(37,0)$ & & & & & \\
\hline \multirow[t]{7}{*}{ Valezi et al. 59 (2014) } & G1 $n=24:$ & $5(20,8)$ & $46,9(5,7)$ & $49(73,1)$ & $30(44,8)$ & & & $27(40,3)$ & $21(31,3)$ \\
\hline & $44,0(10,9)$ & $7(28,0)$ & $47,6(5,7)$ & & & & & & \\
\hline & $\mathrm{G} 2 \mathrm{n}=25$ & $4(17,4)$ & $56,8(5,5)$ & & & & & & \\
\hline & $42,9(9,5)$ & $5(22,7)$ & $46,1(5,4)$ & & & & & & \\
\hline & $\begin{array}{l}\text { G3 n = 23: } \\
43,1(9,5)\end{array}$ & & & & & & & & \\
\hline & G4 n = 22: & & & & & & & & \\
\hline & $43,7(10,7)$ & & & & & & & & \\
\hline Xavier et al. 60 (2010) & $40,5(10,3)$ & $5(25,0)$ & $54,5(7,8)$ & & & & & & \\
\hline
\end{tabular}

DP: desvio padrão; IC95\%: intervalo de 95\% de confiança; IMC: índice de massa corporal $\left(\mathrm{kg} / \mathrm{m}^{2}\right)$.

frequência de pacientes do sexo masculino de $21 \%$ nas amostras. Em média, $21,8 \%$ dos operados eram tabagistas, $60,8 \%$ hipertensos e $22,3 \%$ diabéticos.

A Tabela 5 apresenta ainda a média/porcentagem para cada dado antropométrico e percentual de comorbidades de acordo com os critérios de classificação da qualidade dos estudos - presença de critérios para especificar o que é considerado comorbidade, escore de NOS e escore de Carson. Sete artigos (17,9\%) apresentavam critérios para definir as comorbidades apresentadas, 21 estudos $(53,8 \%)$ foram classificados como melhor qualidade metodológica pelo escore NOS e 15 (38,5\%) foram considerados de melhor qualidade metodológica pelo escore de Carson. Os artigos apresentaram grande heterogeneidade, com mínimo de 50\% para a avaliação do tabagismo e máximo de 97\% para avaliação do
IMC e presença de artropatia. Os resultados de análise de sensibilidade, usando os três escores propostos, confirmaram heterogeneidade em todos os grupos.

\section{Discussão}

A presente revisão sistemática descreveu o perfil de saúde dos pacientes submetidos à cirurgia bariátrica pelo SUS, baseando-se em dados publicados em estudos realizados no Brasil. O SUS ainda não possui um registro com os dados clínicos dos pacientes operados de cirurgia bariátrica em base de dados nacional. A Autorização de Internação Hospitalar (AIH) disponibiliza apenas sexo e idade. Entretanto, informações sobre IMC e comorbidades são essenciais para conhecer o perfil do paciente operado e interpretar dados 
Síntese dos resultados obtidos dos estudos, distribuídos por critério de avaliação utilizado.

\begin{tabular}{|c|c|c|c|c|c|c|c|c|c|c|c|c|c|}
\hline \multirow[t]{2}{*}{ Parâmetro } & \multirow[t]{2}{*}{$N *$} & \multicolumn{3}{|c|}{ Geral } & \multicolumn{3}{|c|}{$\begin{array}{c}\text { Com critério para } \\
\text { descrever comorbidades }\end{array}$} & \multicolumn{3}{|c|}{ NOS $\geq$ média } & \multicolumn{3}{|c|}{ Escore de Carson $\geq$ média } \\
\hline & & Média (\%) & IC95\% & 12 & Média (\%) & $\mathrm{IC} 95 \%$ & 12 & Média (\%) & IC95\% & 12 & Média (\%) & IC95\% & 12 \\
\hline Idade (anos) & 34 & 41,4 & $40,4-42,4$ & 85,4 & 41,5 & $39,2-43,8$ & 91,2 & 42,4 & $41,2-43,6$ & 85,7 & 41,4 & $39,9-42,9$ & 87,1 \\
\hline $\mathrm{IMC}\left(\mathrm{kg} / \mathrm{m}^{2}\right)$ & 35 & 48,6 & $44,5-47,3$ & 96,6 & 49,2 & $47,1-51,3$ & 94,2 & 48,7 & $46,7-50,8$ & 97,6 & 49,3 & $48,3-50,3$ & 84,7 \\
\hline Sexo masculino & 39 & 21,0 & $18,7-23,6$ & 63,5 & 21,1 & $15,1-28,7$ & 84,1 & 20,5 & $17,7-23,6$ & 58,8 & 20,3 & $16,6-24,5$ & 74,4 \\
\hline Hipertensão & 23 & 60,8 & $55,1-62,2$ & 84,4 & 58,9 & $50,0-67,3$ & 80,3 & 64,0 & $57,1-70,4$ & 79,6 & 55,0 & $45,2-64,5$ & 86,3 \\
\hline Diabetes & 23 & 22,3 & $18,5-26,6$ & 79,6 & 23,4 & $18,5-29,2$ & 61,8 & 19,3 & $13,8-26,4$ & 82,2 & 24,1 & $19,6-29,2$ & 74,0 \\
\hline Dislipidemia & 18 & 35,3 & $26,0-45,7$ & 92,3 & 39,2 & $24,0-56,8$ & 93,4 & 31,4 & $22,1-42,5$ & 92,1 & 33,2 & $20,3-48,5$ & 92,1 \\
\hline Artropatia & 6 & 33,7 & $16,3-57,0$ & 97,3 & & & & & & & & & \\
\hline Apneia do sono & 6 & 31,2 & $14,7-54,4$ & 97,3 & & & & & & & & & \\
\hline Tabagismo & 4 & 21,8 & $15,6-29,6$ & 49,5 & & & & & & & & & \\
\hline
\end{tabular}

IC95\%: intervalo de 95\% de confiança; IMC: índice de massa corporal; NOS: Escala Newcastle-Ottawa.

* Número de artigos que contribuíram com a informação.

relacionados a acesso, mortalidade, tempo de internação, entre outros. Esta revisão avança ao prover informações aos interessados em pesquisar sobre o tema e em mostrar as profundas lacunas existentes acerca do conhecimento das condições pré-cirúrgicas do paciente com obesidade grave.

Não existe um padrão ouro para a avaliação da qualidade de estudos observacionais. É sabido que a utilização de escores de qualidade em metanálises de estudos observacionais é controversa, pois os escores construídos de forma pontual podem ter sua validade comprometida e os resultados podem não estar associados necessariamente à qualidade 15,16. O handbook da Cochrane 17 para revisões sistemáticas de estudos não randomizados não recomenda a utilização de qualquer instrumento para a avaliação de qualidade metodológica para esse tipo de estudo e considera que todas as escalas disponíveis necessitam de ajustes para fornecer informações detalhadas sobre viés de seleção, o que é especialmente crítico nesse tipo de revisão.

Apesar do esforço para aferir a qualidade metodológica dos estudos incluídos, houve grande diferença no número daqueles pontuados acima da média, avaliados pela escala de Carson (39\%) ou NOS (54\%). O valor prático dessa diferença não é conhecido, mas a síntese dos resultados obtidos por um ou outro escore não diferiu de forma significativa, mesmo considerando diversos subgrupos. Merece destaque a baixa representatividade numérica das amostras estudadas tanto considerando regiões brasileiras ou, em vários casos, os serviços incluídos no estudo. Além disso, também não podemos excluir que diferentes estudos em um mesmo estado tenham incluído pacientes comuns e, com isto, influenciado o perfil dos pacientes descritos.

Comparando com revisões sistemáticas e artigos clássicos internacionais, os resultados da presente revisão mostraram que os pacientes do SUS apresentam perfil antropométrico e de comorbidades semelhante àqueles incluídos em publicações de outros países 3,18,19,20,21,22, exceto pela elevada prevalência de hipertensão arterial 3,21,23.

Alguns autores sugerem que o número de comorbidades concomitantes seja um preditor de risco aumentado de mortalidade pós-operatória 22,24,25. Buchwald et al. 3 descreveram comorbidades associadas significativamente à mortalidade pós-operatória e incluíram hipertensão, insuficiência cardíaca congestiva, doença cardíaca isquêmica, diabetes, hiperlipidemia, síndrome de hipoventilação, hipertensão pulmonar, doenças hepáticas, comprometimento psicossocial, hérnia abdominal e estado funcional comprometido. Outros autores, em estudos menores, corroboraram esses achados. Hipertensão e diabetes também foram destacados como fatores de risco por Morino et al. 26 .

A comparação dos dados obtidos nesta revisão com pacientes de outros países está sujeita a limitações importantes. Em primeiro lugar, a grande heterogeneidade nos achados pode significar um número pequeno de pacientes incluídos em cada publicação ou até variabilidade aleatória acentuada nas amostras. Em segundo, não há, no mundo, serviço público universal como o 
SUS que ofereça cobertura para 200 milhões de habitantes. Assim, as comparações ficam comprometidas por variáveis não contempladas nas publicações, como nível socioeconômico e educacional dos pacientes, acesso e equidade.

Em síntese, entre os aspectos associados à maior mortalidade ou com maior tempo de internação, a hipertensão está desfavoravelmente presente nas coortes de pacientes assistidos pelo SUS no país, o que pode comprometer o resultado da cirurgia em termos de risco de mortalidade e maior morbidade peri e pós-operatória.

A principal limitação desta revisão foi a baixa qualidade metodológica dos estudos. Além disso, a representação de toda a população submetida à cirurgia bariátrica no Brasil ficou comprometida pelas amostras pequenas incluídas nos estudos, que avaliaram menos de $10 \%$ dos pacientes submetidos a esta cirurgia no Brasil no período. Quando fizemos a análise por região, verificamos que duas regiões do Brasil sequer estão representadas. Também como limitação associa-se o fato de que poucos trabalhos apresentaram os critérios para descrição de comorbidades, o que é um fator adicional de incerteza para o tipo de análise que os estudos propõem. A maioria das publicações provinha de hospitais universitários. Apesar das cirurgias bariátricas pelo SUS serem realizadas também em hospitais públicos, somente escassa informação provém desta fonte. Embora não pareça existir seleção de perfil de comorbidades para a realização de cirurgia em hospital universitário ou hospital público fora do ambiente acadêmico, para uma avaliação mais assertiva seriam necessárias informações de outros centros de atendimento ao paciente bariátrico. Publicações com critérios metodológicos mais estritos são necessárias para a confirmação dos valores encontrados nesta revisão.

\section{Conclusão}

Esta revisão descreveu o perfil antropométrico e de comorbidades do paciente submetido à cirurgia bariátrica pelo SUS. Essa informação não está disponível e é relevante para a avaliação de risco dos pacientes atendidos no sistema público.

O paciente operado no SUS tem perfil de risco e de comorbidades semelhante àquele descrito em estudos internacionais, exceto pela elevada prevalência de hipertensão arterial, o que pode aumentar o risco de desfechos adversos peri e pós-operatórios.

A qualidade metodológica dos artigos recuperados, entretanto, compromete a generalização dos achados desta revisão. A grande amplitude dos intervalos de confiança obtidos na síntese dos dados mostra a imprecisão das informações coletadas. Estudos maiores e com melhor qualidade metodológica são necessários para mais consistência das informações.

\section{Resumen}

En 2013 cerca de un millón de brasileños eran obesos graves. La cirugía bariátrica es una alternativa para la pérdida de peso sostenida y hasta 2014 fueron realizadas 50.000 operaciones por el Sistema Único de Salud de Brasil (SUS), sin embargo, faltan datos antropométricos y de comorbilidades de esos pacientes. El objetivo del estudio fue realizar una revisión sistemática para conocer el perfil del paciente SUS, sometido a la cirugía bariátrica entre 1998-2014. Se consultaron las bases MEDLINE, LILACS, SciELO y Scopus y se evaluó la calidad metodológica de los artículos. Se identificaron 1.591 y se seleccionaron 39; un 95\% eran observaciona- les. En promedio, los pacientes tienen 41,4 años, índice de mas a corporal $48,6 \mathrm{~kg} / \mathrm{m}^{2}$, un $21 \%$ son hombres, un $61 \%$ hipertensos, un $22 \%$ diabéticos y un $31 \%$ presentan apnea del sueño. Comparado con pacientes de los estudios internacionales, el paciente SUS presenta un perfil antropométrico y de comorbilidades semejante, excepto por la mayor prevalencia de hipertensión. La baja calidad metodológica de los estudios sugiere cautela en la interpretación de los resultados.

Cirugía Bariátrica; Derivación Gástrica; Perfil de Salud 


\section{Colaboradores}

S. M. B. Kelles contribuiu com a concepção e projeto, revisão bibliográfica, redação do artigo, revisão crítica do conteúdo e aprovação final. M. F. H. S. Diniz contribuiu com a redação do artigo, revisão crítica do conteúdo e aprovação final da versão a ser publicada. C. J. Machado com a concepção e projeto, revisão do conteúdo e aprovação final da versão a ser publicada. S. M. Barreto contribuiu com a concepção e projeto, revisão crítica do conteúdo intelectual e aprovação final da versão a ser publicada.

\section{Referências}

1. Instituto Brasileiro de Geografia e Estatística. Pesquisa de Orçamentos Familiares 2008-2009: antropometria e estado nutricional de crianças, adolescentes e adultos no Brasil. Brasília: Instituto Brasileiro de Geografia e Estatística; 2009.

2. Reis CEG, Vasconcelos IAL, Barros JFN. Políticas públicas de nutrição para controle da obesidade infantil. Rev Paul Pediatr 2011; 29:625-33.

3. Buchwald H, Avidor Y, Braunwald E, Jensen MD, Pories W, Fahrbach K, et al. Bariatric surgery: a systematic review and meta-analysis. JAMA 2004; 292:1724-37.

4. Ministério da Saúde. Portaria GM/MS no 252, de 30 de março de 1999. Diário Oficial da União 1999; 31 mar.

5. Ministério da Saúde. Portaria GM/MS no 196, de 29 de fevereiro de 2000. Diário Oficial da União 2000; 1 mar.

6. Khawali C, Ferraz MB, Zanella MT, Ferreira SRG Evaluation of quality of life in severely obese patients after bariatric surgery carried out in the public healthcare system. Arq Bras Endocrinol Metabol 2012; 56:33-8

7. Carson CA, Fine MJ, Smith MA, Weissfeld LA, Huber JT, Kapoor WN. Quality of published reports of the prognosis of community-acquired pneumonia. J Gen Intern Med 1994; 9:13-9.

\section{Agradecimentos}

Os autores agradecem à Unimed-BH pelo suporte metodológico para a elaboração da pesquisa e à Fundação de Amparo à Pesquisa do Estado de Minas Gerais, pelo suporte financeiro. Os autores agradecem às bibliotecárias Mariza Cristina Torres Talim e Mariana Fernandes Ribeiro, pela inestimável colaboração para a realização desta revisão sistemática.
8. Wells GA, Shea B, O'Connell D, Peterson J, Welch V, Losos M, et al. The Newcastle-Ottawa Scale (NOS) for assessing the quality if nonrandomized studies in meta-analyses. http://www.ohri.ca/programs/ clinical_epidemiology/oxford.htm (acessado em 30/Ago/2013).

9. Herzog R, Álvarez-Pasquin MJ, Diaz C, Barrio JL, Estrada JM, Gil A. Are healthcare workers' intentions to vaccinate related to their knowledge, beliefs and attitudes? A sistematic review. BMC Public Health 2013; 13:154.

10. Borenstein M, Hedges LV, Higgins JPT, Rothstein HR. Introduction to meta-analysis. Hoboken: John Wiley \& Sons; 2009.

11. Duarte-Guerra LS, Coelho BM, Santo MA, Wang YP Psychiatric disorders among obese patients seeking bariatric surgery: results of structured clinical interviews. Obes Surg 2015; 25:830-7.

12. Moreira RNC, Caetano JA, Barros LM, Galvão MTG. Diagnósticos de enfermagem, fatores relacionados e de risco no pós-operatório de cirurgia bariátrica. Rev Esc Enferm USP 2013; 47:168-75.

13. Oliveira LD, Diniz MT, de Fátima HS, Diniz M Savassi-Rocha AL, Camargos ST, et al. Rhabdomyolysis after bariatric surgery by Roux-en-Y gastric bypass: a prospective study. Obes Surg 2009; 19:1102-7. 
14. Valezi AC, Machado VH. Morphofunctional evaluation of the heart of obese patients before and after bariatric surgery. Obes Surg 2011; 21:1693-7.

15. Stroup DF, Berlin JA, Morton SC, Olkin I, Williamson GD, Rennie D, et al. Meta-analysis of observational studies in epidemiology: a proposal for reporting. Meta-analysis of Observational Studies in Epidemiology (MOOSE) group. JAMA 2008; 283:2008-12.

16. Shamliyan T, Kane RL, Dickinson S. A systematic review of tools used to assess the quality of observational studies that examine incidence or prevalence and risk factors for diseases. J Clin Epidemiol 2010; 63:1061-70.

17. Higgins JPT, Green S. Cochrane handbook for systematic reviews of interventions. Version 5.2.0. http://www.cochrane-handbook.org (acessado em 30/Ago/2013)

18. Sjöström L, Narbro K, Sjöström CD, Karason K, Larsson B, Wedel H, et al. Effects of bariatric surgery on mortality in Swedish obese subjects. N Engl J Med 2007; 357:741-52.

19. Adams TD, Gress RE, Smith SC, Halverson RC, Simper SC, Rosamond WD, et al. Long-term mortality after gastric bypass surgery. N Engl J Med 2007; 357:753-61.

20. Carlin AM, Zeni TM, EnglishWJ, Hawasli AA, Genaw JA, Krause KR, et al. The comparative effectiveness of sleeve gastrectomy, gastric bypass, and adjustable gastric banding procedures for the treatment of morbid obesity. Ann Surg 2013; 257:791-7.

21. Flum DR, Dellinger EP. Impact of gastric bypass operation on survival: a population-based analysis. J Am Coll Surg 2004; 199:543-51.

22. Kelles SMB, Barreto SM, Guerra HL. Mortality and hospital stay after bariatric surgery in 2,167 patients: influence of the surgeon expertise. Obes Surg 2009; 19:1228-35.

23. Chang SH, Stoll CR, Song J, Varela JE, Eagon CJ, Colditz GA. The effectiveness and risks of bariatric surgery: an updated systematic review and metaanalysis, 2003-2012. JAMA Surg 2014; 149:275-87.

24. DeMaria EJ, Murr M, Byrne TK, Blackstone R, Grant JP, Budak A, et al. Validation of the obesity surgery mortality risk score in a multicenter study proves it stratifies mortality risk in patients undergoing gastric bypass for morbid obesity. Ann Surg 2007; 246:578-82.

25. Ferraz EM, Martins Filho ED, Ferraz AAB. Avaliação dos fatores de risco pré-operatórios. In: Diniz MTC, Diniz MFHS, Sanches SRA, Savassi-Rocha $\mathrm{AL}$, editores. Cirurgia bariátrica e metabólica: abordagem multidisciplinar. São Paulo: Editora Atheneu; 2012. p. 71-3.

26. Morino M, Toppino M, Forestieri P, Angrisani L, Allaix ME, Scopinaro N. Mortality after bariatric surgery: analysis of 13,871 morbidly obese patients from a national registry. Ann Surg 2007; 246:10027.

27. Abreu MR, Ramos AP, Vendramini RC, Brunetti IL, Pepato MT. Steatosis and hepatic markers before and shortly after bariatric surgery. Ann Clin Biochem 2007; 44(Pt 1):63-9.
28. Anderi E, Araújo LGC, Fuhro FE, Godinho CA, Henriques AC. Experiência inicial do serviço de cirurgia bariátrica da Faculdade de Medicina do ABC. Arq Méd ABC 2007; 32:25-9.

29. Blume CA, Boni CC, Casagrande DS, Rizzolli J, Padoin AV, Mottin CC. Nutritional profile of patients before and after Roux-en-Y gastric bypass: 3-year follow-up. Obes Surg 2012: 22:1676-85.

30. Cardozo DD, Hilgert JB, Hashizume LN, Stein AT, Souto KE, Meinhardt NG, et al.Impact of bariatric surgery on the oral health of patients with morbid obesity. Obes Surg 2014; 24:1812-6.

31. Carneiro G, Flório RTB, Zanella MT, Pradella-Hallinan M, Ribeiro-Filho FF, Tufik S, et al. Is mandatory screening for obstructive sleep apnea with polysomnography in all severely obese patients indicated? Sleep Breath 2012; 16:163-8.

32. Cazzo E, Jimenez LS, Pareja JC, Chaim EA. Effect of Roux-en-Y gastric bypass on nonalcoholic fatty liver disease evaluated through NAFLD fibrosis score: a prospective study. Obes Surg 2015; 25:982-5.

33. Costa RCN, Yamaguchi N, Santo MA, Riccioppo D, Pinto-Junior PE. Outcomes on quality of life, weight loss and comorbidities after Roux-en-Y gastric bypass. Arq Gastroenterol 2014; 51:165-70.

34. Costa-Matos A, Guidoni LR, Carvalho KA, Fernandes RC, Perez MD. Is there an association between urolithiasis and Roux-en-Y gastric bypass surgery? Int Braz J Urol 2009; 35:432-5.

35. Cunha LC, Cunha CL, Souza AM, Chiminacio Neto N, Pereira RS, Suplicy HL. Evolutive echocardiographic study of the structural and functional heart alterations in obese individuals after bariatric surgery. Arq Bras Cardiol 2006; 87:615-22.

36. Diniz MF, Passos VM, Barreto SM, Linares DB, de Almeida SR, Rocha AL, et al. Different criteria for assessment of Roux-en-Y gastric bypass success: does only weight matter? Obes Surg 2009; 19:1384-92.

37. Donadelli SP, Salgado Jr. W, Marchini JS, Schmidt A, Amato CA, Ceneviva R, et al. Change in predicted 10-year cardiovascular risk following Roux-enY gastric bypass surgery: who benefits? Obes Surg 2011; 21:569-73.

38. Franca FT. Avaliação da perda de peso e da qualidade de vida de pacientes obesos mórbidos submetidos à cirurgia de bypass gastric em Y de Roux com anel redutor e sem anel, após o primeiro ano de seguimento [Monografia]. São Paulo: Hospital do Servidor Público Municipal de São Paulo; 2011.

39. Freire RH, Borges MC, Alvarez-Leite JI, Correia MITD. Food quality, physical activity, and nutritional follow-up as determinant of weight regain after Roux-en-Y gastric bypass. Nutrition 2012; 28:53-8.

40. Freitas CRB. Efeito da perda de peso induzida pela cirurgia bariátrica sobre a função respiratória [Dissertação de Mestrado]. Natal: Programa de Pósgraduação em Fisioterapia, Universidade Federal do Rio Grande do Norte; 2011.

41. Freitas Junior WR, Ilias EJ, Kassab P, Cordts R, Porto PG, Rodrigues FCM, et al. Assessment of the body composition and the loss of fat-free mass through bioelectric impedance analysis in patients who underwent open bariatic bypass. Scient World J 2014; 2014:843253. 
42. Jóia Neto L, Lopes-Junior AG, Jacob CE. Alterações metabólicas e digestivas no pós-operatório de cirurgia bariátrica. ABCD Arq Bras Cir Dig 2010; 23:266-9.

43. Kuga R, Safatle-Ribeiro AV, Faintuch J, Ishida RK, Furuya Jr. CK, Garrido AB, et al. Endoscopic findings in the excluded stomach after Roux-en-Y gastric bypass surgery. Arch Surg 2007; 142:942-6.

44. Laurino Neto RM, Herbella FA, Tauil RM, Silva FS, de Lima Jr. SE. Comorbidities remission after Roux-en-Y gastric bypass for morbid obesity is sustained in a long-term follow-up and correlates with weight regain. Obes Surg 2012; 22:1580-5.

45. Lima MLRP, Mourão SCO, Diniz MTC, Leite VHR. Hepatic histopathology of patients with morbid obesity submitted to gastric bypass. Obes Surg 2005; 15:661-9.

46. Machado CE. Estudo sobre a compulsão alimentar em pacientes submetidos à cirurgia bariátrica [Dissertação de Mestrado]. São Paulo: Faculdade de Medicina, Universidade de São Paulo; 2008.

47. Moreira MA, Silva SA, Araújo CM, Nascimento CC. Clinical-nutritional evaluation of obese patients submitted to Roux-en-Y gastric bypass. Acta Gastroenterol Latinoam 2010; 40:244-50.

48. Oliveira IV. Cirurgia bariátrica no âmbito do Sistema Único de Saúde: tendências, custos e complicações [Dissertação de Mestrado]. Brasília: Universidade de Brasília; 2007.

49. Paisani DM, Chiavegato LD, Faresin SM. Volumes, capacidades pulmonares e força muscular respiratória no pós-operatório de gastroplastia. J Bras Pneumol 2005; 31:125-32.

50. Pedrosa IV, Burgos MGPA, Souza NC, Morais CN. Aspectos nutricionais em obesos antes e após a cirurgia bariátrica. Rev Col Bras Cir 2009; 36:316-22.

51.Pinheiro-Junior S, Pinhel MAS, Nakazone MA, Pinheiro A, Amorim GFS, Florim GMS, et al. Effect of genetic variants related to lipid metabolism as risk factors for cholelithiasis after bariatric surgery in Brazilian population. Obes Surg 2012; 22:623-33.
52. Porto MFM. Gastroplastia redutora no Hospital do Servidor Público Municipal. Avaliação de perda de peso e complicações - cinco anos de seguimento [Monografia]. São Paulo: Hospital do Servidor Público Municipal de São Paulo; 2012.

53. Salgado Jr. W, Cunha FQ, dos Santos JS, NoninoBorges CB, Sankarankutty AK, de Castro e Silva Jr. O. Routine abdominal drains after Roux-en-Y gastric bypass: a prospective evaluation of the inflammatory response. Surg Obes Relat Dis 2010; 6:648-52.

54. Salgado Jr. W, Rosa GV, Nonino-Borges CB, Ceneviva R. Prospective and randomized comparison of two techniques of staple line reinforcement during open Roux-en-Y gastric bypass: oversewing and bioabsorbable Seamguard. J Laparoendosc Adv Surg Tech Part A 2011; 21:579-82.

55. Scabim VM, Eluf-Neto J, Tess BH. Adesão ao seguimento nutricional ambulatorial pós-cirurgia bariátrica e fatores associados. Rev Nutr 2012; 25:497-506.

56. Silva AMO, Boin FS, Pareja JC, Magna LA. Análise da função respiratória em pacientes obesos submetidos à operação FobiCapella. Rev Col Bras Cir 2007; 34:314-20.

57. Silva EN, Sanches MD. Perfil lipídico de obesos antes e após a derivação gástrica a Fobi-Capella. Rev Col Bras Cir 2006; 33:91-5.

58. Silva MAM, Rivera IR, Barbosa EMW, Crispim MAC, Farias GC, Fontan AJA, et al. Frequência de fatores de risco cardiovascular antes e 6 e 12 meses após gastroplastia. Rev Assoc Med Bras 2013; 59:381-6.

59. Valezi AC, Marson AC, Merguizo RA, Costa FL. Roux-en-Y gatric bypass: limb length and weight loss. ABCD Arq Bras Cir Dig 2014; 27 Suppl 1:56-8.

60. Xavier MAF, Ceneviva R, Terra Filho J, Sankarankutty AK. Pulmonary function and quality of life in patients with morbid obesity six months after bariatric surgery. Acta Cir Bras 2010; 25:407-15.

Recebido em 14/Fev/2014

Versão final reapresentada em 27/Abr/2015 Aprovado em 27/Mai/2015 